\title{
Dynamic VISI Methods For OLSE and Syndrome Calculation using Synchronized Mitigation Procedures for Intact Circuit Functionality
}

\author{
Farha Anjum, P.Keerthana, Kavya Reddy, Kiran, Samhitha
}

\begin{abstract}
Now a days in VLSI design circuit's reliability has become the major parameter of concern. With the consistently expanding requests for higher speed and lower control correspondence frameworks, productive VLSI executions of those blunder redress codes have extraordinary significance for reasonable applications.

There exists various synchronized moderation procedures proposed to ensure that the blunders don't influence the circuit usefulness. Among them, to ensure the recollections and registers in electronic circuits Error Correction Codes (ECC) is normally utilized. At whatever point any ECC method is utilized, the encoder and decoder circuit may likewise endure mistakes. Here synchronized slip identification Also revision method to OLS encoders (OLSE) What's more syndrome figuring is suggested What's more assessed. Those suggested technique proficiently executes An equality prediction plan that detects the greater part errors that influence An solitary out hub utilizing the properties of OLS codes. Today VLSI design means usage of Verilog or VHDL. In this research work Verilog HDL is used for simulation and Synplify for synthesis purpose.
\end{abstract}

Index terms : VLSI, SRAM, VHDL

\section{INTRODUCTION}

As VLSI innovation scales into the nanometer space, VLSI frameworks are liable to progressively pervasive cataclysmic deformities, delicate blunders, and critical parametric varieties [4]. For information theory and more coding theory with provisions done programming building and networking transmission, botch area Also change alternately screw up control would methods that enable robust movement for electronic data over deceitful correspondence channels.

Revised Manuscript Received on July 05, 2019.

Dr. Farha Anjum, Professor in ECE Dept at Siddhartha Institute of Engineering and Technology, Hyderabad, India

P.Keerthana, UG Student, ECE Dept at Siddhartha Institute of Engineering and Technology, Hyderabad, India

Kavya Reddy, UG Student, ECE Dept at Siddhartha Institute of Engineering and Technology, Hyderabad, India
Various correspondence channels would at risk to channel upheaval Furthermore in this manner mistakes may a chance to be exhibited Throughout transmission starting with the hotspot to An beneficiary. Screw up ID number techniques tolerance distinguishing such mistakes, same time screw up amendment empowers revamping of the initial data Likewise An lead. The dependability of recollections relies upon measurements of the circuit, working voltages, and coordinated thickness Triple secluded excess codes and mistake amending codes are for the most part utilized for blunder revising incorporation densities, it expands the quantity of delicate mistakes, which needs higher mistake remedy abilities. Some multi mistake bit amendments Codes are reed Solomon codes and Bose-Chaudhuri-Hocquenghem codes, however in which the calculation is so perplexing and it is cycle based. The decoders to interpret in fixed rate thus it lessen the working criteria. To achieve higher capacity to recognize blunders among mistake adjusting codes the sub gathering of low thickness equality check code called semi cyclic LDPC codes has been chosen to expand the exhibition of the decoders to distinguish and address enormous number of mistakes. It has a place with group of dominant part rationale disentangling.

The purpose behind utilizing ML disentangling is that it is extremely easy to execute and commonsense and has low multifaceted nature. Turbo-codes with execution close to the Shannon limit point of confinement have gotten impressive consideration since their presentation in 1993 [1][2]. Ideal usage methodologies of turbo codes are still of high intrigue, especially since turbo codes have turned into a standard for $3 \mathrm{G}$. VLSI consecutive structures of turbo decoders comprise of $\mathrm{M}$ Soft-Input Soft-Output (SISO) decoders, either associated in a pipeline, or autonomously handling their very own encoded squares [3][4][5]. The two designs process $M$ turbo squares all the while and are proportionate as far as coding gain, throughput, inertness and multifaceted nature. For the unraveling of enormous square sizes, successive

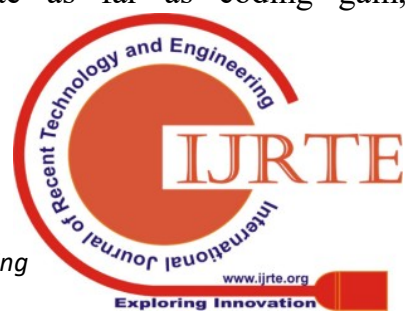


structures require huge measure of memory per SISO for $\mathrm{M}$ turbo squares stockpiling. Thus, improving throughput by copying SISOs is territory wasteful. Likewise, inertness is high because of iterative unraveling, making the consecutive engineering unacceptable for inactivity delicate applications, for example, versatile interchanges, intuitive video and telemedicine.

Restricted to easier inactivity will be to decrease those amount for obliged deciphering iterations, Be that that might corrupt the coding addition. A intriguing treestructured SISO methodology [6] altogether lessens those latency, at those expense of an expanded zone prerequisite. Parallel deciphering schemes [7][8] perform the SISO sliding window algorithm utilizing An number for sub-block SISOs to parallel, every preparing a standout amongst the sliding windows.

\subsection{Error correction codes}

Today's innovation scaling goes with impacts concerning both control utilization and unwavering quality. The paper introduced here bargains for the blending of both parts. A standout amongst the unwavering quality issues need aid single off chance impacts (SEE) because of radiation. Officially in the 70's, memory phones were liable for investigations about radiation prompted lapse rates [1], and nowadays, SEEs need aid of worry to static memories [2], latches Also flipflops [3] Furthermore actually for irregular combinational rationale $[4,5]$. Dependent upon now, an expansive mixed bag about solidifying systems need been recommended to those different sorts from claiming structures. As those delicate lapse rate (SER) about memory components On irregular rationale is ceaselessly expanding [6] What's more Concerning illustration the measure from claiming flip-flops What's more latches will be quickly growing, this paper may be helping of the security about these capacity components. Also the secondary defenselessness will SEEs, scaling prompts an expanded energy thickness for chip which Disallows a recurrence build Concerning illustration seen in the secret word.

The excellent low energy configuration strategies bring at present expanding importance $[7,8]$, Be that as must make complemented Eventually Tom's perusing massine parallelism [9] Furthermore control management clinched alongside networks Also frameworks for a chip. Same time energy gating may be employed, whether modules will be unused for An instead in length time for time, There is an assortment about insurance schemes against single-event bombshells (SEU) to flip-flops accessible [10], every one from claiming them present redundancy, extra action What's more extra force utilization. Noteworthy Advance need likewise been settled on should limit this energy expand by exceptional outlines in BISER [20], razor [12], DF-DICE [21] also how. However, these schemes don't target those clock gated phase, which is really By and large a bigger time period An state must a chance to be held over those clocked period. An right on time deliberate utilization of lapse identification might have been Toward jewish scribes in the exact duplicate of the jewish bible, starting when christ. To hardware Also computing, a delicate lapse is An sort of slip the place a sign isn't right. Errors might be brought about Toward a defect, generally error On configuration alternately development or An broken part. There are two sorts for delicate errors, chip-level delicate lapse What's more system-level delicate lapse. When those radioactive iotas in the chip's material rot What's more discharge alpha particles under the chip consequently Chip-level delicate errors happen. Though it is detected, a delicate slip might be remedied by changing right information set up of slip information. Exceptionally dependable frameworks utilize slip revision on right delicate errors on the fly. Handy correspondence may be also utilized to electronic units by slip identification Furthermore lapse revision. It lessens the level for commotion What's more interferences done electronic medium. Sign on clamor proportion is the measure for slip identification and revision and its viability.

\subsection{Overview}

Since huge numbers years, to identifying Also correcting errors lapse revision Codes (ECCs) were utilized. Analysts need suggested extensive variety from claiming codes to memory requisitions. To correcting you quit offering on that one bit for every word, absolute slip revision (SEC) codes are utilized within general. Propelled codes which can likewise right twofold contiguous errors need aid also been contemplated. Lapse correcting codes are an focal and only mossycup oak current correspondence frameworks. Those best known such codes - best in the sense from claiming performing closest of the hypothetical Shannon cutoff - would turbo codes [1], low-thickness equality check codes [2], [3] and varieties thereof. Such codes would decoded Toward "probability propagation"- An form of the non specific sum-product calculation [4] or varieties thereof. Brief late reviews about such codes and their deciphering are [5] and [6] separately. As opposed will large portions different sign preparing errands On a interchanges receiver, the deciphering for lapse correcting codes need constantly been executed digitally.

It is normal that these additions can add up to two requests of extent [9]-[11]. Fundamentally the same as simple decoders were researched by Hagenauer at al [12][14]. Elective ways to deal with simple disentangling incorporate the simple Viterbi decoders of [15]-[18]; for further references see [9]. Unmistakably, the presentation of such simple decoders will be influenced by a wide range of non idealities. Most such impacts are not effectively evaluated. On a fundamental level, every single such impact could be contemplated by SPICE-level Monte-Carlo recreations, yet such reenactments are awfully tedious to be very useful. Better techniques are certainly required. However, the issue with some mind boggling codes that revises more mistakes is commonly restricted by their effect on postponement and power, which thusly will confine their appropriateness to memory plan. To run-over those worries, a procedure is proposed by the utilization of codes that are One Step Majority Logic Decodable (OSMLD). One Step Majority Logic Decodable codes are lowidleness decodable codes. In this way, for ensuring recollections, they are utilized. The other sort of

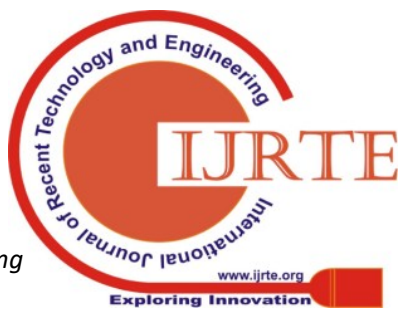


code that is One Step Majority Logic Decodable is Orthogonal Latin Squares (OLS) code. For interconnections, recollections, and stores utilization of OLS codes have picked up a recharged intrigue, in light of the particularity with the end goal that mistake adjusting capacities can be effectively adjusted to the blunder rate or to the method of activity.

Regularly greater equality bits are required for OLS codes than different codes for revising a similar number of blunders. Be that as it may, because of their measured quality and the basic, low postpone unraveling usage, kill this detriment in numerous applications. This proposal is given to the proficient VLSI engineering plan and execution for mistake remedying coding. Today, the mistake rectifying coding has turned out to be one vital part in about all the advanced information transmission and capacity frameworks. With the persistently expanding requests for higher speed and lower control correspondence frameworks, upgraded VLSI executions of those blunder redressing codes that are at present utilized in useful applications have incredible current significance. Since limited fields are broadly utilized in numerous prevalent mistake amending codes, e.g., ReedSolomon (RS) codes, the productive equipment usage of limited field number juggling units are significant for those coding framework executions. Inferable from the broad uses of RS codes in reality, RS codec VLSI execution has gotten colossal considerations and will stay to be a vivacious research theme for quite a while.

Moreover, examination of the VLSI usage to other developing Furthermore All the more capable errorcorrecting codes may be exceedingly alluring and greatly critical to the improvement for next-generation information transmission and capacity frameworks. The as of late rediscovered low-thickness Parity-Check (LDPC) codes accepted enormous attentions be- reason for their fantastic error-correcting proficiency What's more fully parallel deciphering algorithm that will prompt highsounding deciphering. A greater amount recently, summed up low-thickness (GLD) Parity-Check codes, the regulate generalization about LDPC codes, were suggested Similarly as guaranteeing elective of the item code to large portions genuine provisions.

To brief, for SRAM memories Furthermore caches, those insurance of the encoders Also syndrome calculation for OLS codes would viewed as. Contingent upon a few positive properties, it is exhibited that equality prediction may be an profitable system to identify What's more right errors in the encoder What's more syndrome calculation. To mossycup oak piece codes it is not those the event for which equality prediction won't furnish viable security. So, it is favorable element from claiming OLS codes furthermore should its modularity Furthermore lowdecoding proficience.

Dissimilar screw up changing codes could make used depend upon the properties of the skeleton and the requisition the place the misstep redressing is to a chance to be introduced. All around slip Correcting Codes would described under square codes Furthermore convolution codes. For the two codes the dissimilar component to those request may be the closeness alternately nonattendance for memory in the encoders. To prepare An square code, those approaching information stream may be divided under squares Also each square is took care of only by including overabundance as for every An proposed computation.

The decoder forms each square separately and rectify blunders by misusing excess. Huge numbers of the significant square codes utilized for mistake recognition are cyclic codes. These are likewise called cyclic repetition check codes.

To a convolutional code, those encoding operation could a chance to be seen Likewise the individuals discrete - time convolution of the enter course of action to those drive response of the encoder. Those compass of the drive response equals the individuals memory of the encoder. Accordingly, the encoder with a convolution code meets expectations on the approaching message sequence, using An "sliding window" climb to to compass will its identity or memory.

Consequently in a convolutional code, Dissimilar to An piece code the place code expressions would handled with respect to a piece Eventually Tom's perusing square basis, those channel encoder acknowledges message odds as constant grouping What's more thereby generates a constant succession of encoded odds toward An higher rate.

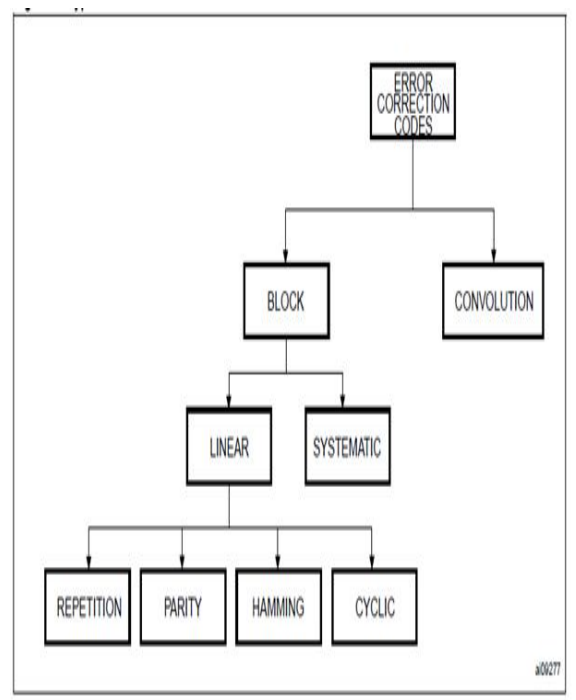

Fig1.2.1: Types of error correction codes

\section{CONCLUSION}

For detecting and correcting errors Error Correction Codes were used. There are different types of codes some of them are EG codes, Reed-Solomon and Hamming codes. Protection of encoder and decoder circuits needed the use of ECCs. A portion complex codes that right All the more errors need aid by and large set by their effect around delay Also power, which thus will farthest point their relevance to memory plans. Will run-over the individuals concerns, An procedure may be suggested Toward the utilization from claiming codes that would OLS codes. For the vast majority of the square codes equality expectation won't give compelling assurance. Along these lines, it is a preferred position of OLS codes notwithstanding its
\& Sciences Publication

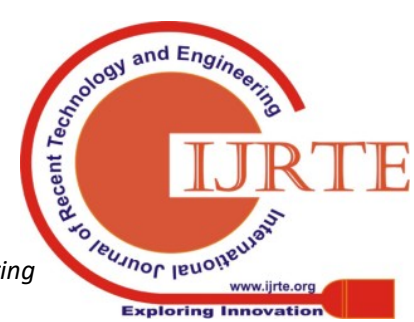


seclusion and low-interpreting ability. In that the territory overhead of keeping away from rationale sharing was $35 \%$. The liability of the checker will be moreover greater to hamming. In this particular case, those every last bit crazy overhead to the recommended arrangement might make through $80 \%$. This affirms the recommended framework isn't forcing clinched alongside an all case and more relies on the properties of OLS codes on fulfill an proficient use.

\section{References}

1. DR. Anubhuti Khare, Manish Saxena, Jagdish Patel "FPGA Implementation of Viterbi Decoder.

2. Jushwanth Xavier. Benujah. B.R, "Multiple bits upset deduction /correction for memory application" in IJAIS., February 2013, pp .15-18.

3. Eiji Fujiwara, code design for Dependable Systems: Theory and practical Application. New York: Wiley, 2006.

4. E. J. Mc Cluskey, "Design techniques for testable embedded error checkers", IEEE Computer, vol.23, no.7, pp.84-88, Jul.1990.

5. Lin Shu, Shu Lin and Daniel. J. Costello, Error Control Coding, $2^{\text {nd }}$. Englewood Cliffs, NJ: PrenticeHall, 2004.

6. S. Ghosh and P .D. Lincoln, "Dynamic low-density parity check codes for fault tolerant nano scale memory", in Proc. Found. Nanosci., 2007, pp.1-5.

7. Salvatore Pontarelli, G.C. Cardarelli, M. Ottavi M. Re , and A. Salsano, "Fault tolerant solid state mass memory for space applications", IEEE Trans.Aerosp.Electron. Syst., vol.41, no.4, pp.13531372, Oct. 2005

8. H. Naeimi and A. DeHon "Corrections to Fault secure Encoder and Decoder for Nano Memory Applications"

9. M. Y. Hsiao, D. Bossen, and R. T. Chein, "Orthogonal Latin square codes," IBM J. Res. Develop., vol. 14, no. 4, pp.390-394, Jul.1970.

10. S. E. Lee, Y. S. Yang, resilent interconnection with Orthogonal Latin squares", IEEE Design Test Computation., vol.28, no.2, pp.30-39, Mar.-Apr.2011. 